\title{
Aged Cattle Brain Displays Alzheimer's Disease-Like Pathology and Promotes Brain Amyloidosis in a Transgenic Animal Model
}

\section{OPEN ACCESS}

Edited by:

Nitton Custodio,

Peruvian Institute of Neurosciences

(IPN), Peru

Reviewed by:

Diana Laura Castillo-Carranza,

University of Monterrey, Mexico

M. Heather West Greenlee,

lowa State University, United States

*Correspondence:

Ines Moreno-Gonzalez

inesmoreno@uma.es

Claudio Soto

Claudio.Soto@uth.tmc.edu

Specialty section:

This article was submitted to Alzheimer's Disease and Related

Dementias,

a section of the journal

Frontiers in Aging Neuroscience

Received: 15 November 2021

Accepted: 17 December 2021

Published: 31 January 2022

Citation:

Moreno-Gonzalez I, Edwards G III,

Morales R, Duran-Aniotz C, Escobedo

G Jr, Marquez M, Pumarola M and

Soto C (2022) Aged Cattle Brain

Displays Alzheimer's Disease-Like

Pathology and Promotes Brain

Amyloidosis in a Transgenic Animal

Model.

Front. Aging Neurosci. 13:815361

doi: 10.3389/fnagi.2021.815361

\begin{abstract}
Ines Moreno-Gonzalez ${ }^{1,2,3,4 *}$, George Edwards III' ${ }^{1}$, Rodrigo Morales ${ }^{1,4}$, Claudia Duran-Aniotz ${ }^{1,5,6}$, Gabriel Escobedo Jr. ${ }^{1}$, Mercedes Marquez ${ }^{7}$, Marti Pumarola ${ }^{7,8}$ and Claudio Soto ${ }^{1 *}$

${ }^{1}$ Department of Neurology, Mitchell Center for Alzheimer's Disease and Related Brain Disorders, University of Texas Health Science Center at Houston, Houston, TX, United States, ${ }^{2}$ Departamento Biología Celular, Genética y Fisiología, Instituto de Investigacion Biomedica de Malaga-IBIMA, Universidad de Malaga, Malaga, Spain, ${ }^{3}$ Center for Biomedical Research on Neurodegenerative Diseases (CIBERNED), Madrid, Spain, ${ }^{4}$ Centro Integrativo de Biologia y Quimica Aplicada (CIBQA), Universidad Bernardo O'Higgins, Santiago, Chile, ${ }^{5}$ Center for Social and Cognitive Neuroscience (CSCN), School of Psychology, Universidad Adolfo Ibáñez, Santiago, Chile, ${ }^{6}$ Latin American Institute for Brain Health (BrainLat), Universidad Adolfo Ibanez, Santiago, Chile, ${ }^{7}$ Department of Animal Medicine and Surgery, Veterinary Faculty, Animal Tissue Bank of Catalunya (BTAC), Universitat Autònoma de Barcelona, Bellaterra (Cerdanyola del Valles), Barcelona, Spain, ${ }^{8}$ Networking Research Center on Bioengineering, Biomaterials and Nanomedicine (CIBER-BBN), Universitat Autonoma de Barcelona, Bellaterra (Cerdanyola del Valles), Barcelona, Spain
\end{abstract}

Alzheimer's disease (AD) is one of the leading causes of dementia in late life. Although the cause of $A D$ neurodegenerative changes is not fully understood, extensive evidence suggests that the misfolding, aggregation and cerebral accumulation of amyloid beta $(A \beta)$ and tau proteins are hallmark events. Recent reports have shown that protein misfolding and aggregation can be induced by administration of small quantities of preformed aggregates, following a similar principle by which prion diseases can be transmitted by infection. In the past few years, many of the typical properties that characterize prions as infectious agents were also shown in $A \beta$ aggregates. Interestingly, prion diseases affect not only humans, but also various species of mammals, and it has been demonstrated that infectious prions present in animal tissues, particularly cattle affected by bovine spongiform encephalopathy (BSE), can infect humans. It has been reported that protein deposits resembling $A \beta$ amyloid plaques are present in the brain of several aged non-human mammals, including monkeys, bears, dogs, and cheetahs. In this study, we investigated the presence of $A \beta$ aggregates in the brain of aged cattle, their similarities with the protein deposits observed in $A D$ patients, and their capability to promote $A D$ pathological features when intracerebrally inoculated into transgenic animal models of AD. Our data show that aged cattle can develop AD-like neuropathological abnormalities, including amyloid plaques, as studied histologically. Importantly, cow-derived aggregates accelerate $A \beta$ amyloid deposition in the brain of AD transgenic animals. Surprisingly, the rate of induction produced by administration of the cattle material was substantially higher than induction produced by injection of similar amounts of human AD material. Our findings demonstrate that cows develop seeding-competent $A \beta$ aggregates, similarly as observed in AD patients.

Keywords: amyloid, prions, Alzheimer's disease, spreading, protein misfolding, seeding, cattle 


\section{INTRODUCTION}

Alzheimer's disease (AD) is the most common form of dementia among elderly people and one of leading public health problems in developed countries. $\mathrm{AD}$ involves progressive brain atrophy, neuronal death, synaptic dysfunction, astrogliosis and the accumulation of protein aggregates in the form of amyloidbeta $(\mathrm{A} \beta)$ deposits, and tau neurofibrillary tangles. Although the etiology of $\mathrm{AD}$ is not yet clear, extensive evidence suggests that the central pathological event is the misfolding, aggregation and brain deposition of $A \beta$ and tau (Soto, 2003; Huang and Mucke, 2012; Masters and Selkoe, 2012). Amyloid accumulates as senile plaques and diffuse deposits in the brain parenchyma and around cerebral blood vessels walls termed cerebral amyloid angiopathy (CAA) (Gomez-Isla et al., 2008).

In addition to $\mathrm{AD}$, various other protein misfolding diseases (PMDs) are thought to be caused by accumulation of misfolded aggregated proteins in various tissues, including highly prevalent illnesses such as Parkinson's disease (PD), type 2 diabetes and more than 20 other diseases (Chiti et al., 2006; Soto, 2012). Among the latter, prion diseases are quite intriguing because they are transmissible by infection through a proteinaceous infectious agent known as prion (Prusiner, 1998). The molecular mechanism responsible for prion infectivity depends on the ability of the misfolded prion protein aggregates to act as seeds, inducing the templated conversion of natively folded prion protein into the developing aggregates (Soto, 2012). In this manner, the pathological protein progressively grows by recruiting more and more of the normal protein. This process is often referred as seeding/nucleation polymerization. Importantly, protein aggregation of all proteins involved in PMDs follows the seeding-nucleation mechanism (Caughey and Lansbury, 2003; Soto, 2012). The similarities between prion replication and amyloid formation, and the intrinsic ability of aggregated seeds to self-propagate the polymerization process led us and others to hypothesize over 10 years ago that misfolded aggregates associated to other PMDs can spread by the prion principle (Soto et al., 2006; Walker et al., 2006). Remarkably, a series of exciting recent reports have demonstrated that several PMDs can be experimentally transmitted by a prionlike mechanism in various cellular and animal models of diverse diseases (Prusiner, 2012; Walker and Jucker, 2015). Over the past decade, many of the hallmark properties of prions as infectious agents have been shown to be shared by several of the prionlike misfolded proteins. For the specific case of $A \beta$, studies from us and other groups have shown that inoculation of transgenic mouse models of amyloidosis with tissue homogenates from patients affected by $\mathrm{AD}$ results in induction or acceleration of amyloid pathology in the recipient animals (Kane et al., 2000; Meyer-Luehmann et al., 2006; Morales et al., 2011; Watts et al., 2011). Moreover, in animals not genetically programmed to develop the disease spontaneously, inoculation with $\mathrm{AD}$ brain homogenates leads to a completely de novo disease, more akin to infectious prions (Morales et al., 2011; Rosen et al., 2011). Importantly, pathological induction can be reduced by depleting the inoculum of $\mathrm{A} \beta$ aggregates (Meyer-Luehmann et al., 2006; Duran-Aniotz et al., 2014). Even more strikingly, efficient induction has been observed by the addition of misfolded protein aggregates prepared in vitro using synthetic $\mathrm{A} \beta$ (Stöhr et al., 2012). Accumulation of $A \beta$ aggregates can be promoted by inoculation of very small amounts of aggregated seeds (Fritschi et al., 2014; Morales et al., 2015a) and titration experiments have shown that the rate of induction is proportional to the amount of seeds inoculated. Finally, disease transmission has been observed even when seeds were administered systemically (Eisele et al., 2010).

The findings described above suggest that $A \beta$ and other misfolded protein aggregates can indeed behave as prions. Still, the main controversy is whether other misfolded proteins can act as infectious agents to transmit the disease among individuals under natural conditions (Fernández-Borges et al., 2013; Irwin et al., 2013; Beekes et al., 2014; Collinge, 2016). Aside from this important point, another aspect that has not been explored is the possibility that protein aggregates accumulating in animals may initiate the disease in humans. Indeed, prion diseases affect not only humans, but also various species of mammals. The accumulation of $A \beta$ aggregates has not been extensively analyzed in animals, however, it has been reported that $\mathrm{AD}$ aggregates are present in the brain of several aged non-human mammals, including monkeys, bears, dogs, and cheetahs (Moreno-Gonzalez and Soto, 2018). In bovine brains, $A \beta$ has been as previously observed as granular aggregates, but not depositing in plaques (Costassa et al., 2016). In this study, we investigated the presence of AD-like pathology in aged cow brains and whether, in analogy to prion diseases, $A \beta$ aggregates derived from cattle brain can induce $\mathrm{A} \beta$ misfolding and aggregation in a transgenic mice model of $\mathrm{AD}$ amyloidosis.

\section{MATERIALS AND METHODS}

\section{Cattle Samples}

Cattle samples were obtained from the Animal Tissue Bank of Catalunya (BTAC), Department of Animal Medicine and Surgery, Veterinary Faculty, Universitat Autonoma de Barcelona, Bellaterra (Cerdanyola del Valles), Barcelona, Spain. Brain samples were obtained from slaughterhouses after the animals were sacrificed. Samples with a post-mortem time lower than $10 \mathrm{~h}$ were immersed in formol, processed and embedded in paraffin. As shown in Supplementary Figure 1, we received 63 samples from female cattle that were 13 to 23 years old. These animals were from 10 different breeds, including Charolais, Bruna of Pirineus, Pirinenca, Limousin, Montbeliard, Simmental, and crossbreeds. We also obtained ten samples of 10 month-old young crossbreed calves that were used as controls. Samples were obtained from the temporal area and contained the hippocampal area, entorhinal cortex, and part of the thalamus.

\section{Human Samples}

AD brain hippocampal samples were acquired from the National Disease Research Interchange (USA). Informed consent was obtained for experimentation with human subjects. The Code of Ethics of the World Medical Association (Declaration of Helsinki) was followed to perform research on human samples and they were manipulated following the universal precautions 
for working with human samples and as directed by the Institutional Review Board of McGovern Medical School at The University of Texas Health Science Center at Houston.

\section{Brain Homogenate}

Cattle and human brain tissue were homogenized at $10 \% \mathrm{w} / \mathrm{v}$ in PBS containing a cocktail of protease inhibitors for western blot and ELISA quantifications. For intracerebral inoculations, samples were homogenized at $40 \% \mathrm{w} / \mathrm{v}$ in the same solution and sterilized by the addition of $1 \%$ of antibiotic/antimycotic solution (Gibco) and $\gamma$-irradiated for $1 \mathrm{~h}$.

\section{Western Blot}

$10 \%$ cattle brain homogenates were mixed with denaturing loading buffer (Invitrogen), heated for $10 \mathrm{~min}$ at $95{ }^{\circ} \mathrm{C}$ and fractionated in $4-12 \%$ NuPAGE gels (Invitrogen). Proteins were transferred to a nitrocellulose membrane (GE Healthcare), blocked with $10 \%$ milk, and incubated with rabbit anti-A $\beta 42$ polyclonal antibody (Covance). After incubation with secondary antibody, $A \beta 42$ was visualized by chemoluminescence using ECL plus (GE Healthcare) in a dark chamber (BioRad).

\section{ELISA}

Mouse brain hemispheres were homogenized at 10\% w/v in PBS containing a cocktail of protease inhibitors. Brain extracts were centrifuged at 32,600 rpm for $1 \mathrm{~h}$ at $4{ }^{\circ} \mathrm{C}$ in an ultracentrifuge (Beckman-Coulter). The pellets were resuspended in $200 \mu \mathrm{L}$ of $70 \%$ formic acid followed by sonication. Samples were centrifuged for $30 \mathrm{~min}$ in the same conditions and the supernatant was collected. This insoluble fraction was neutralized in $1 \mathrm{M}$ Tris buffer, $\mathrm{pH} 11$. Brain levels of $\mathrm{A} \beta 42$ were measured using a human $\mathrm{A} \beta$ ELISA kit (Invitrogen) on an ELISA plate reader (EL800 BIO-TEK).

\section{Histology}

Serial $10-\mu$ m-thick sections from all cattle, human, and mouse groups were processed for immunostaining. After blocking the endogenous peroxidase activity with $3 \% \quad \mathrm{H}_{2} \mathrm{O}_{2}-10 \%$ methanol for $20 \mathrm{~min}$, sections were incubated overnight at room temperature in mouse anti-A $\beta 4 \mathrm{G} 8$ (1:1,000 Covance). Sections stained for $A \beta$ were pretreated with $85 \%$ formic acid. Primary antibody was detected by incubating $1 \mathrm{~h}$ with goat anti-mouse HRP-linked secondary antibody and the peroxidase reaction was visualized using a DAB Kit (Vector) following the manufacturer's instructions. For counterstaining, sections were incubated in Harris hematoxylin for $1 \mathrm{~min}$ (Fisher). For Thioflavin-S (ThS) staining, tissue slices were incubated in ThS solution $(0.025 \%$ in $50 \%$ ethanol) for $8 \mathrm{~min}$. Finally, all sections were dehydrated in graded ethanol, cleared in xylene, coverslipped with DPX mounting medium, and examined under a bright field/epifluorescent microscope (DMI6000B, Leica).

\section{Animals}

Hemizygous APP/PS1 (B6C3-Tg APPswe, PSEN1dE9 85Dbo/J, The Jackson Laboratory) mice express human amyloid precursor protein (APP695swe) and a mutant human presenilin 1 (PS1dE9) in a B6C3 background. These animals develop amyloid plaques and other AD-like features starting around 6 months of age (Jankowsky et al., 2004). Animals were housed in groups of up to five in individually ventilated cages under standard conditions $\left(22^{\circ} \mathrm{C}, 12 \mathrm{~h}\right.$ light-dark cycle) receiving food and water ad libitum. All animal experiments were carried out in accordance with the $\mathrm{NIH}$ regulations and approved by the committee of animal use for research at The University of Texas Health Science Center at Houston. Mice were sacrificed by $\mathrm{CO}_{2}$ inhalation and perfused transcardially with PBS. Brains were removed, post-fixed into fixative solution (10\% neutral buffered formalin) and embedded in paraffin.

\section{Animal Inoculation}

For intracerebral inoculation, 30-40 days-old APP/PS1 mice were injected with $10 \mu \mathrm{L}$ of $40 \%(\mathrm{w} / \mathrm{v})$ cattle or human brain homogenate in each hemisphere ( $n=8$ to 11 per group), without any purification or isolation. Briefly, mice were anesthetized using isoflurane. Skin was incised and a small hole was drilled in the skull and samples were injected into both hippocampi. At the end of the treatment, skin was closed using surgical suture. Animals were placed on a thermal pad until recovery and monitored daily for several days.

\section{Histological Quantification}

Burden quantification was done through the lateromedial extent of the cortical and hippocampal areas in the sagittal plane, being the first section of each animal randomly collected. Quantification was assessed in every tenth section (with a distance of $100 \mu \mathrm{m}$ among them), and four to six $10 \mu \mathrm{m}$ sections were measured for each animal ( $n=8-11$ per group). Photomicrographs were taken by using a DFC310 FX Leica digital camera, imported into Image $1.45 \mathrm{~s}$ software (NIH) and converted to black and white images. Threshold intensity was manually set and kept constant, and the number of pixels was determined for $4 \mathrm{G} 8$ immunostained sections to quantify amyloid load in the hippocampal formation (CA1, CA2, CA3, and dentate gyrus) and several cortical areas (including motor, somatosensory, visual, frontal, parietal and retrosplenial cortex). Analysis for each was done by a single examiner blinded to sample identities.

\section{Statistical Analysis}

Graphs are expressed as means \pm standard error (s.e.m.). After confirming normal distribution with Skewness and Kurtosis statistic test, $T$-test or one way analysis of variance (ANOVA) followed by a post-hoc Tukey's multiple comparisons test were used to analyze differences among groups. Statistical analyses were performed using GraphPad Prism 5.0 software (GraphPad Software Inc). Statistical differences for all tests were considered significant at the $p<0.05$ level.

\section{RESULTS}

To investigate whether cattle develops AD-related abnormalities, we performed a detailed histological study for the presence of amyloid-like deposits in the brain of cows of different ages. The majority of the samples were from female cattle of the Bruna of Pirineus and various crossbreed 
(Supplementary Figure 1A). Old animal ages ranged from 13 to 23 years old (Supplementary Figure 1B). For this study, we analyzed the hippocampus, temporal cortex, and thalamus of more than 60 cows (Supplementary Figure 1C). For most of the specimens analyzed, we had access to both snap-frozen and formalin-fixed material. Positive and negative controls included human $\mathrm{AD}$ brains and 10 month-old calf brains, respectively. Paraffin embedded fixed tissue from all the specimens (cattle and human samples) were processed and stained for $A \beta$. The amino acid sequence of $A \beta$ in both humans and cattle is exactly the same (Johnstone et al., 1991); therefore, we were able to use the same antibodies. After a complete histopathological analysis, we observed a scattered appearance of $\mathrm{A} \beta$ deposits in many slices of some aged cow brains (Figure 1A). A $\beta$ deposits were detected in the cortex and hippocampus of various cows $>13$ years old, but not in any young animals. Cow amyloid deposits were reactive against human anti-A $\beta$ antibody (4G8), depositing as mature plaques similar to those seen in human $\mathrm{AD}$ patients' brains (Figure 1A, left panels). We also observed perivascular deposits, which are highly reminiscent of cerebral amyloid angiopathy lesions commonly seen in human $\mathrm{AD}$ brains (Figure 1A, center panels). In addition, intracellular $A \beta$ staining was observed in both cattle and human brains (Figure 1A, right panels). Most of the deposits observed in cattle brain corresponded to fibrillar amyloid aggregates, as confirmed by the positive staining of the plaques using thioflavin-S (ThS) in the consecutive slice (Figure 1B). In total, 14 out of 63 brains from cows older than 13 years of age displayed $A \beta$ aggregates in the brain areas studied, although just nine of them presented $A \beta$ plaques similar to those observed in humans (14.3\%). To examine the relative amount of amyloid deposits observed in cattle brain and compare it with that present in patients affected by $\mathrm{AD}$, we performed image analysis of the temporal area from 14 old cows exhibiting amyloid pathology and five patients with AD (Figure 1C). The results show that the brains of old cows have a substantial amount of amyloid deposits. Indeed, in the hippocampal region, the animals analyzed showed an amyloid burden of $0.37 \pm$ $0.07 \%$, which means that $0.37 \%$ of the brain area analyzed was occupied by amyloid. Nevertheless, this burden is significantly smaller ( $>10$-fold lower) than the one estimated on the $\mathrm{AD}$ patients $(3.82 \pm 1.0 \%)$ analyzed (Figure 1C). We could not detect any $\mathrm{A} \beta$ aggregates in young cattle brain samples.

We and others have shown that inoculation of $A \beta$-rich brain homogenate from $\mathrm{AD}$ patients or transgenic mice is able to induce an early $\mathrm{AD}$-like pathology in recipient transgenic animals (Meyer-Luehmann et al., 2006; Eisele et al., 2010; Morales et al., 2011). Knowing that the sequence of cattle $\mathrm{A} \beta$ peptide is identical to the human one, we wanted to test whether cattle brain harboring amyloid aggregates exacerbate AD pathology in susceptible mice. To that end, we intracerebrally (i.c.) injected amyloid-containing cow brain tissue extracts into a double transgenic (APP/PS1) mouse model of brain amyloidosis (Jankowsky et al., 2004) and analyzed the possible acceleration of the pathology. For the experiment, the following five groups $(n=$ 8-11 per group) of animals were used: (i) the experimental group injected with $40 \%$ brain homogenate from an old cow containing amyloid deposits (Old cow/A $\beta+$ ); (ii) a group injected with the

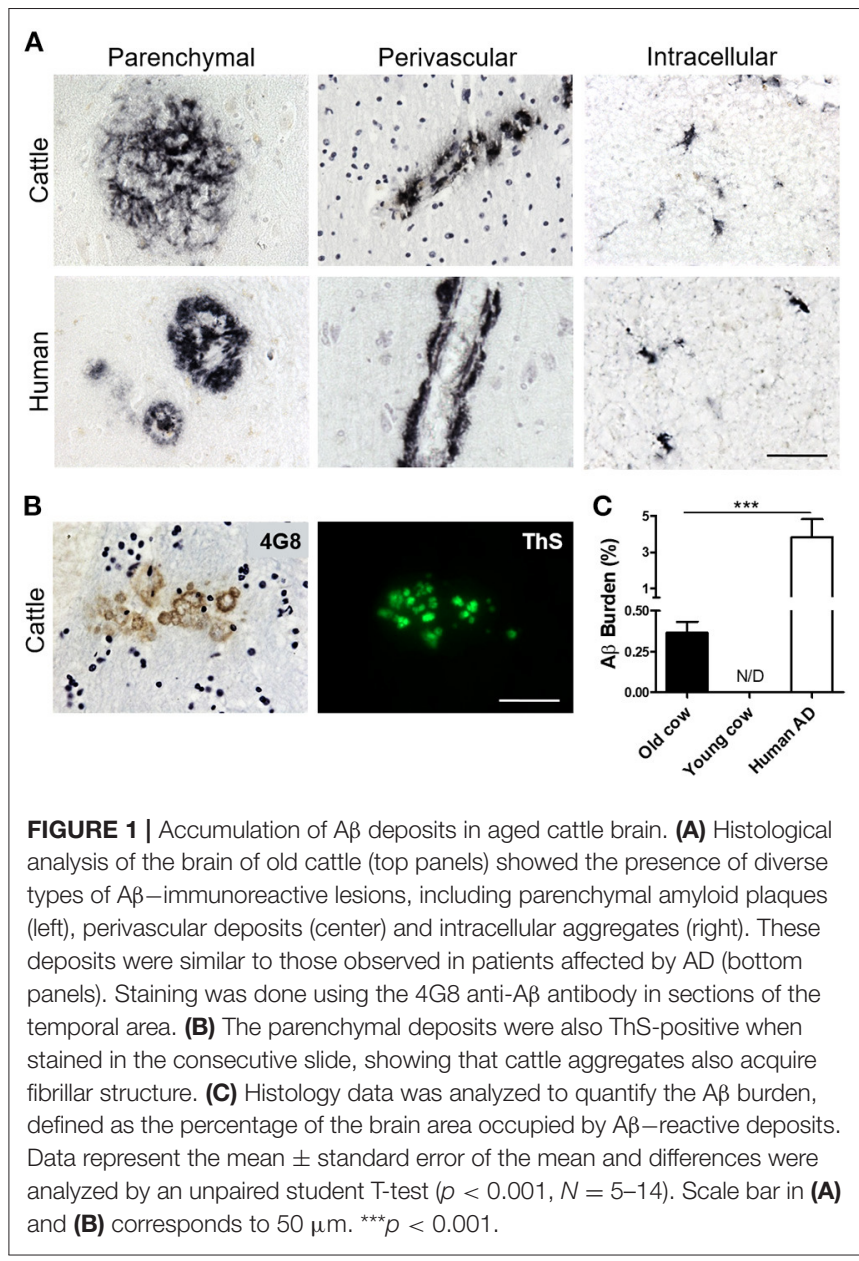

same amount of old cow brain homogenate in which amyloid deposition was not detected by histological or biochemical analysis (old cow/A $\beta-$ ); (iii) a control group injected with $40 \%$ brain extract from a young cow; (iv) a negative control consisting of untreated animals; and (v) a positive control in animals injected with human $\mathrm{AD}$ brain homogenate. Before treatment, the inocula for the different groups were thoroughly analyzed by histological and biochemical techniques to measure the presence and quantity of $A \beta$ aggregates. Figure $2 \mathrm{~A}$ shows the comparative histological staining of representative brain sections from the different subjects used for inoculation. No amyloid staining was seen in any of the slices analyzed from the young cow or the old cow $/ A \beta-$. In contrast, the old cow $/ A \beta+$ exhibited scattered appearance of $4 \mathrm{G} 8$ positive deposits reminiscent of amyloid plaques, similar in morphology to human $\mathrm{AD}$ brains (Figure 2A). Biochemical analysis of cattle brain homogenate by western blot demonstrated that the specimen selected for the experimental group (old cow $1 / \mathrm{A} \beta+$ ) presented a wide range of high molecular weight species reactive with an antibody that recognizes specifically human A $\beta 42$ (Figure 2B). Only some of these bands were detectable and at a much lesser intensity in 10month-old young cow or the old cows/A $\beta-$, used as age-matched controls. The samples indicated with an asterisk in Figure 2B 


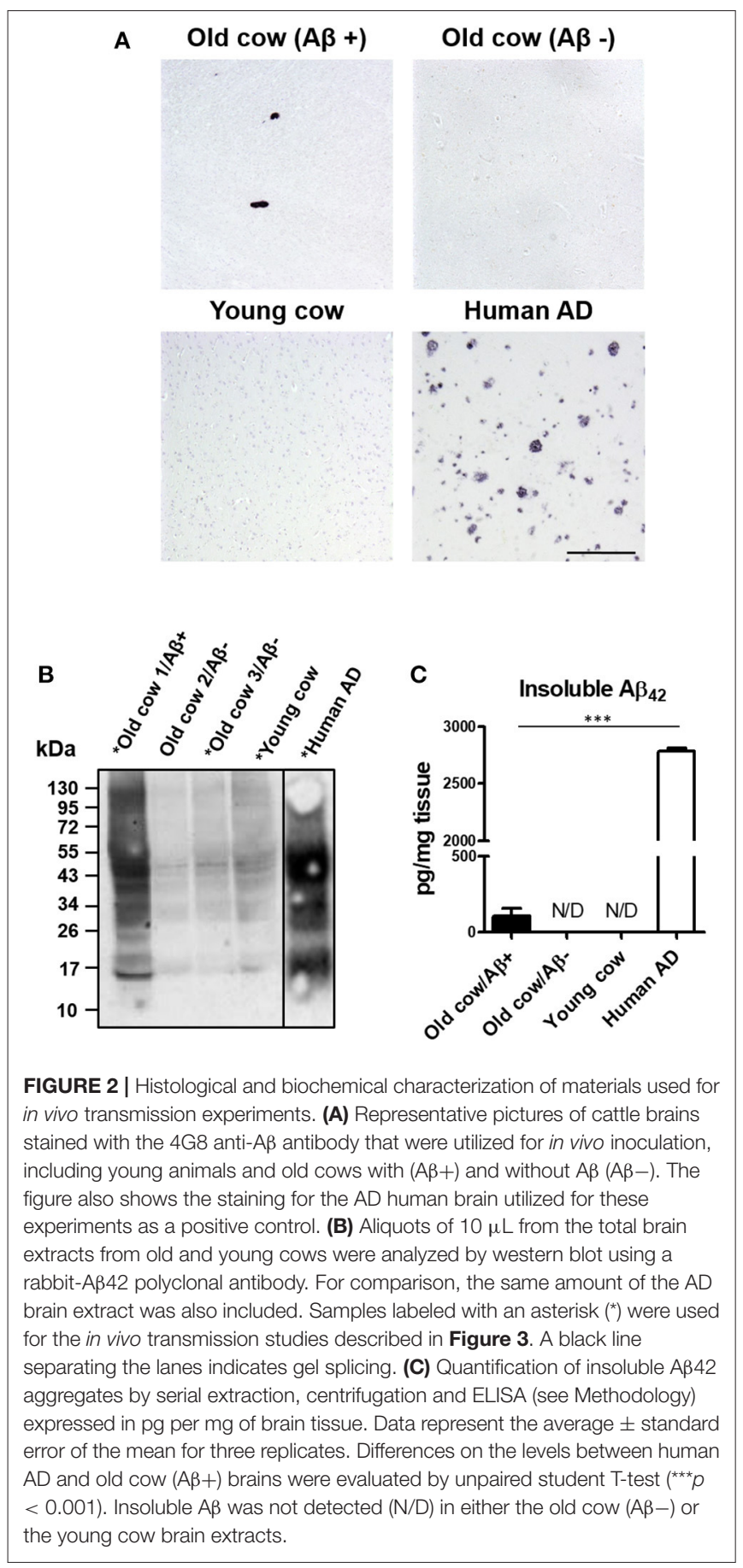

were used for the transmissibility experiments described below. Finally, we measured the amount of $\mathrm{A} \beta 42$ in the insoluble fraction after extraction with formic acid in each of the inocula (Figure 2C). The results showed a concentration of insoluble $\mathrm{A} \beta 42$ of $31.72 \mathrm{pg} / \mathrm{mg}$ of tissue in the old cow/A $\beta+$. In contrast, no detectable insoluble $A \beta 42$ was observed in the young cow or the old cow/A $\beta-$. It is important to highlight that even though the levels of insoluble $A \beta 42$ in the old cow/A $\beta+$ brain were significantly higher than in young animals, they represent only around $1 \%$ of the levels detectable in human $\mathrm{AD}$ brain homogenate $(2,788.78 \mathrm{pg} / \mathrm{mg})$ (Figure 2C).

To evaluate the ability of $A \beta$ aggregates deposited in cattle brain to seed amyloid formation in vivo, double transgenic animals were injected i.c. at 30-40 days old with $10 \mu \mathrm{L}$ of $40 \%$ amyloid-containing old cow brain homogenate into both hippocampal hemispheres through stereotactic surgery $(\mathrm{Tg}+$ Old cow/A $\beta+)$. In addition, various groups of control animals were treated with the diverse inocula described above. Animals were sacrificed at 6 months-old, when they normally start developing plaques due to the transgenic expression of the mutant genes. 4G8 immunostaining showed that animals injected with amyloid-containing cattle brain homogenate displayed more amyloid plaques in the cortical area than the control groups (Figure 3A, top panels). When $A \beta$ load was measured by image analysis, we could observe that there was a statistically significant increase in $A \beta$ burden (stained area/total area analyzed) in the group injected with old cow/A $\beta+$. The increase was as much as 2 -fold $(0.24 \pm 0.04$ vs. 0.11 $\pm 0.03 \%$ ) compared to non-treated animals (Figure 3B). Analysis of $\mathrm{A} \beta$ deposition in the hippocampus, where the inoculation occurred, showed similar results (Figure 3A, bottom panels). Quantification of the hippocampal amyloid burden in experimental and control groups also showed that animals injected with $\mathrm{A} \beta$-containing cattle brain homogenate doubled the regular amount of $\mathrm{A} \beta(0.11 \pm 0.02$ vs. $0.05 \pm 0.01 \%)$ found in the hippocampus of APP/PS1 at this age (Figure 3C). Moreover, staining of fibrillar aggregates by $\mathrm{ThS}$ also showed that animals injected with $A \beta$-containing cow brain tissue displayed more ThS-positive plaques in both cortex and hippocampus than control groups (Figure 4). These results indicate that amyloid deposits present in cattle brain homogenate contain seeding-competent $A \beta$ aggregates that are able to accelerate $A \beta$ deposition when inoculated into a transgenic animal model of AD.

To compare the seeding capability of cattle and human $A \beta$ aggregates to induce amyloid deposition, APP/PS1 transgenic animals were also inoculated with human brain extracts from a patient affected by $\mathrm{AD}$. The amyloid deposition pattern and burden was analyzed by immunohistochemistry in both groups. As shown in Figure 5A, the human inoculum was able to induce amyloid deposition to a substantially higher extent than the cattle inoculum in both cortex and hippocampus. In the cortical area, the human brain injection increases amyloid burden 4 -fold more than the cattle inoculum $(0.92 \pm 0.22$ vs. $0.24 \pm 0.04 \%)$ (Figure 5B, $p=0.003 T$-test), whereas in the hippocampus the increase was $\sim 10$-fold $(1.59 \pm 0.30$ vs. $0.15 \pm 0.2 \%$ ) (Figure $5 C$, $p<0.0001 T$-test). However, when the induction ratio (amyloid burden/concentration of insoluble $\mathrm{A} \beta 42$ injected) was calculated, the data indicates that cattle material promotes amyloid deposition $>10$-fold better than an equivalent quantity of human $A \beta$ aggregates (Figures 5D,E). This surprising data suggests that although the cattle sample contains less concentration of aggregated $A \beta$, these structures appear to be more competent to seed amyloid deposition than human samples. In addition, the human aggregates produced a higher proportion of smaller 


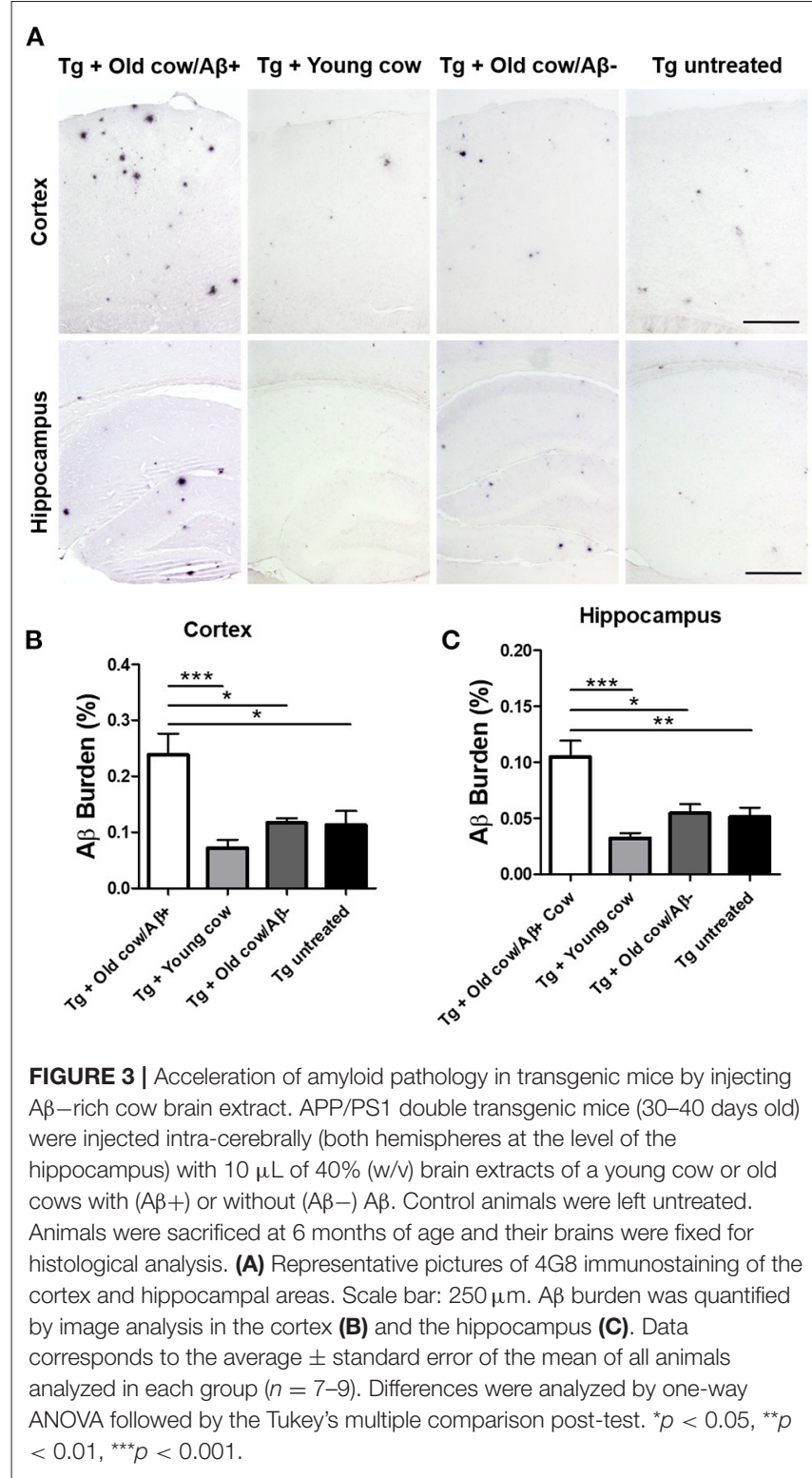

parenchymal and perivascular amyloid aggregates and in areas where plaques are not generally found in the non-treated animal, such as in the corpus callosum (Figure 5A), whereas cow seeds seem to trigger the formation of larger plaques. The differences in the seeding competency and the profile of aggregates induced suggest that $A \beta$ aggregates present in human and cattle brain may represent different arrangements or "conformational strains" of $\mathrm{A} \beta$ seeds.

\section{DISCUSSION}

The prion-like induction and spreading of misfolded protein aggregates implicated in several protein misfolding disorders is a recently recognized process with potentially important implications to understand the etiology and progression of

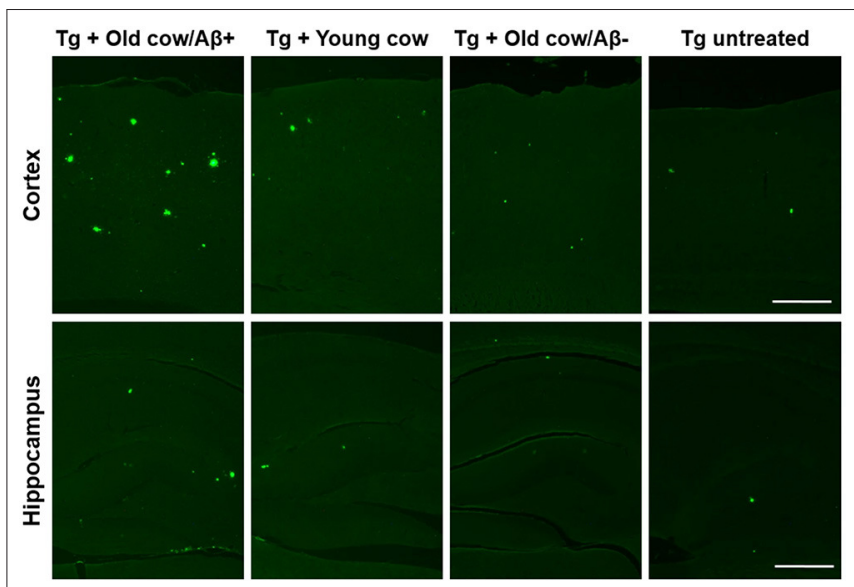

FIGURE 4 | A $\beta$-rich cattle tissue induced high amounts of fibrillar plaques. Representative pictures of ThS staining in APP/PS1 transgenic mice inoculated with brain extracts from $A \beta$-containing old cattle, old cow with no detectable $A \beta$ aggregates, and young cattle. For a negative control, we used transgenic mice left untreated. Scale bar: $250 \mu \mathrm{m}$.

these diseases and the development of novel strategies for therapeutic intervention (Prusiner, 2012; Soto, 2012; Walker and Jucker, 2015). The prion principle of disease transmission posits that a misfolded protein aggregate is able to transfer biological information by converting the normal form of the protein into more of the abnormal, disease-associated form. This process initiates the progressive generation of misfolded protein aggregates that spread to other areas of the brain and accumulate over time to produce brain damage and disease. The prionlike transmission of the pathological folding-operating at the molecular and cellular levels-is probably at the root of the spreading of damage throughout the brain that is characteristic of neurodegenerative diseases (Moreno-Gonzalez and Soto, 2011; Thal et al., 2014; Walker and Jucker, 2015). However, in prion diseases transmission also occurs among different individuals in which prions act as bona fide infectious agents to spread disease in animal or human populations. Moreover, in the case of prions the disease can be transmitted across animals from different species (Béringue et al., 2008), with the landmark case being the generation of a new human disease, termed variant CreutzfeldtJakob disease, produced by exposure to cattle affected by bovine spongiform encephalopathy (Collinge et al., 1996; Bruce et al., 1997; Scott et al., 1999). Evidence gathered over the past 10 years has demonstrated that both protein aggregates implicated in $\mathrm{AD}$ (A $\beta$ and tau) can propagate under experimental conditions as prions (Prusiner, 2012; Soto, 2012; Morales et al., 2015a,b; Walker and Jucker, 2015).

The main goal of our study was to investigate whether cattle present $\mathrm{AD}$-like lesions in the brain, and if these pathological features display prion-like seeding activity. For this purpose, we characterized the brains from many cows at different ages for the presence of amyloid deposits and subsequently the ability of those aggregates to induce amyloid pathology in a mouse model of amyloid deposition. The results of this study show 


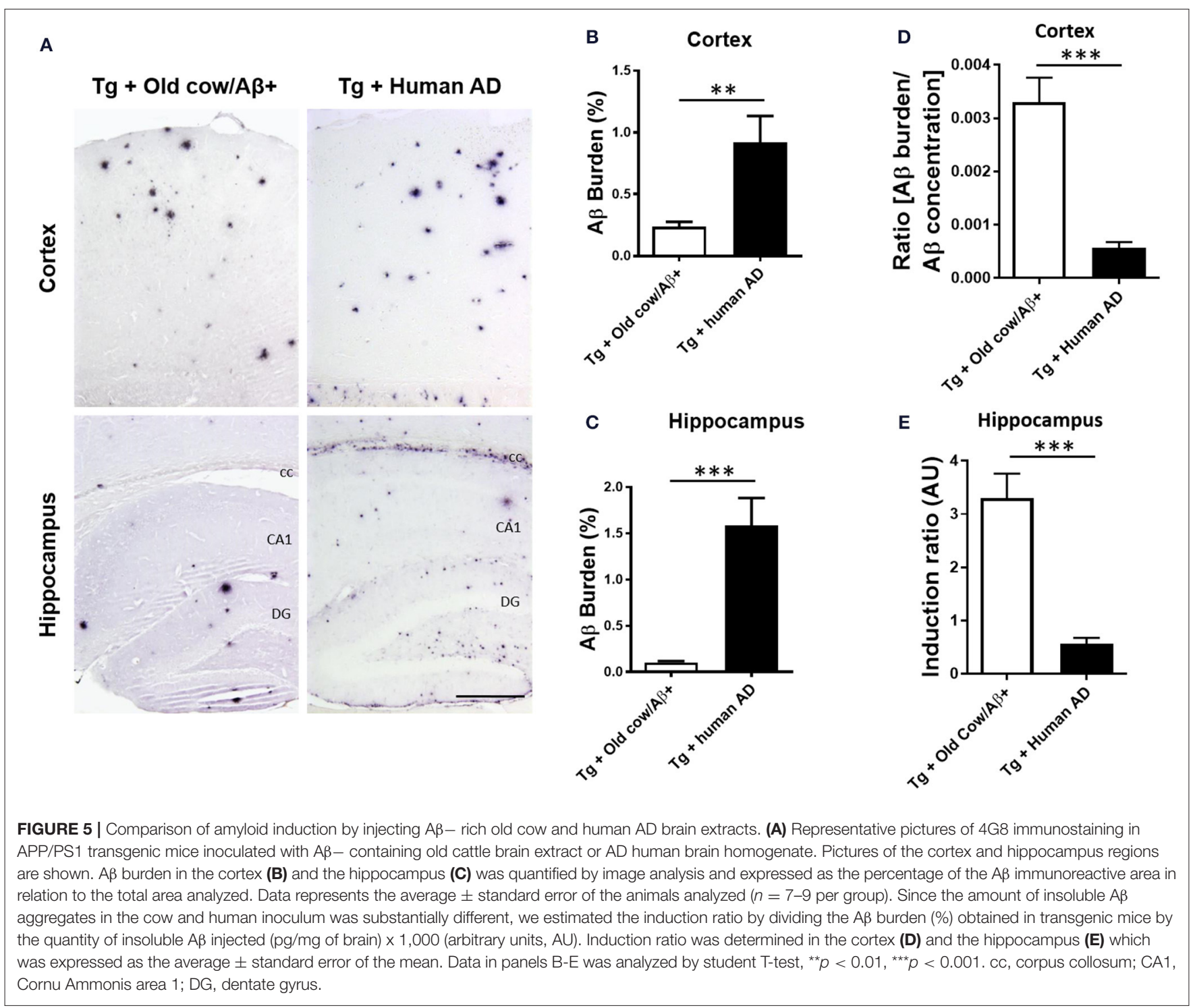

that some old cows spontaneously develop amyloid deposits in their brain. Interestingly, these deposits were similar to those present in human cases of $\mathrm{AD}$. Out of the 63 cattle brain samples analyzed, nine of them (14.3\%) displayed typical $A \beta$ aggregates in the analyzed areas. Therefore, appearance of amyloid deposits in cattle brain appears at a frequency comparable to the prevalence of $\mathrm{AD}$ pathological abnormalities in humans, since it is estimated that $\mathrm{AD}$ affects about $6 \%$ of people 65 years and older, and the incidence doubles every 5 years. Of course, many more elderly non-demented people display amyloid pathology in their brains, a condition that is usually considered as preclinical or prodromal AD (Tagliavini et al., 1988; Price et al., 2009).

Cattle brains were also analyzed for hyperphosphorylated tau (ptau) and tangle formation. We found that only three specimens had elevated levels of ptau using AT8 antibody. These brains were not used as inoculum for the intracerebral inoculation study to specifically evaluate the effect of $A \beta$ aggregates. We cannot exclude that brains could also contain other types of amyloid seeds including tau oligomers that we were unable to detect. Although tau aggregates can modulate $\mathrm{A} \beta$ toxicity, it has been reported that they are not able to induce $A \beta$ aggregation (Bloom, 2014; Nisbet et al., 2015). This, together with the use of APP mice, allows evaluating the seeding capability of $A \beta$ using complete brain homogenates, excluding the effect of potential other seeds present in the inoculum.

Although behavioral or cognitive abnormalities were not analyzed in cows, the amount of $A \beta$ deposits observed parallels pre-clinical $\mathrm{AD}$. Importantly, brain extracts from old cows containing $A \beta$ aggregates were able to significantly increase amyloid deposition when injected intra-cerebrally into a double transgenic mouse model of $\mathrm{AD}$ amyloidosis. Though the total 
level of pathological induction was lower than the one produced by inoculation of $\mathrm{AD}$ brain homogenates, the cow material showed a higher promotion activity than human tissue when the rate of induction was corrected by the amount of $A \beta$ aggregates injected. This surprising result suggests that cattle aggregates are better seeds than their human counterparts. The most likely explanation for this result is that cattle deposits are smaller, less compact and contain smaller amounts of other components compared to human aggregates, which presumably remain deposited in the brain for much longer periods of time. This interpretation is supported by our previous observations showing that brain tissue from people affected by mild cognitive impairment (MCI), which is considered a pre-clinical form of $\mathrm{AD}$, produced significantly higher $\mathrm{A} \beta$ induction than tissue from established AD patients (Duran-Aniotz et al., 2013). Moreover, Jucker and colleagues have shown that small, soluble $\mathrm{A} \beta$ oligomers are better seeds in vivo than large fibrillar aggregates (Langer et al., 2011). We have recently published data demonstrating that $\mathrm{AD}$ patients displaying different amyloid pathology induce different pathological features in the same transgenic models used in this study (Duran-Aniotz et al., 2021). In that line, the distinctive pathology observed between cattle and human $\mathrm{AD}$ patients may be responsible of their dissimilar seeding activity. It could well be that these differences are due to the fact that cattle develop different $\operatorname{strain}(\mathrm{s})$ of $\mathrm{A} \beta$, which are able to accelerate aggregation with smaller amounts of the original seeds or more efficiently. A more in-depth study would be needed to determine the presence of a different $A \beta$ strain in cattle.

The prion-like transmission of $\mathrm{A} \beta$ aggregates has been extensively reported in animal models and likely plays an important role in the progressive spreading of pathological abnormalities throughout the brain (Moreno-Gonzalez and Soto, 2011; Thal et al., 2014; Walker and Jucker, 2015). Nevertheless, whether this phenomenon ever operates in the inter-individual transmission of disease pathology in humans remains highly debatable. Recent studies have provided evidence for the induction of $A \beta$ aggregation in people receiving human pituitaryderived growth hormone (Jaunmuktane et al., 2015; Ritchie et al., 2017). However, when the risk of $\mathrm{AD}$ development, and not only amyloid pathology, was studied no evidence was found for disease transmission (Irwin et al., 2013). The findings of our current study suggest that $A \beta$ aggregates present in the brains of old cattle are competent to seed amyloid deposition in vivo. This induction has also been observed with other protein aggregates such as AA amyloid (Rising et al., 2021). However, the potential transmission of $A \beta$ cattlederived seeds to humans is unlikely, considering that repeated oral administration of $\mathrm{AD}$ brain extracts to susceptible mice failed to accelerate pathological features (Morales et al., 2021). The results presented in this manuscript suggest that aged

\section{REFERENCES}

Beekes, M., Thomzig, A., Schulz-Schaeffer, W. J., and Burger, R. (2014). Is there a risk of prion-like disease transmission by Alzheimer- or cattle are susceptible to develop pathological features similar to $\mathrm{AD}$, and that misfolded $\mathrm{A} \beta$ present in their brain is seeding competent.

\section{DATA AVAILABILITY STATEMENT}

The raw data supporting the conclusions of this article will be made available by the authors on reasonable request.

\section{ETHICS STATEMENT}

The studies involving human participants were reviewed and approved by Institutional Review Board, The University of Texas Health Science Center at Houston. Informed consent was obtained by the National Disease Research Interchange (NDRI). The animal study was reviewed and approved by Committee of Animal Use for Research, The University of Texas Health Science Center at Houston.

\section{AUTHOR CONTRIBUTIONS}

IM-G designed the experiments, participated in animal manipulation, performed the histological, biochemical, image, and statistical analyses, prepared the figures, and wrote the manuscript. GEIII participated in animal manipulation and performed the histological analysis. RM participated in animal manipulation and participated in the experimental design. CD-A performed histological, image, and statistical analysis. GEJr participated in histological analysis. MM and MP provided cattle samples and performed their initial neuropathological analysis. CS supervised the entire project, designed the research plan, and wrote the manuscript. All authors read and approved the final manuscript.

\section{FUNDING}

This work was partially funded by the Alzheimer's Association (NIRP-12-257323 to IM-G, AARGD-18-566576 to RM), the Spanish Ministry of Science and Innovation PID2019107090RA-I00 and Ramon y Cajal Program RYC-2017-21879 (IM-G), the National Institutes of Health RF1AG072491 (RM) and 3P01AI077774-09S1 to CS, and an award from the Mitchell Foundation (CS) 2018-AARG-591107, ANID/FONDEF ID20I10152, ANID/FONDECYT 1210622, and Anillo ACT210096 to CD-A.

\section{SUPPLEMENTARY MATERIAL}

The Supplementary Material for this article can be found online at: https://www.frontiersin.org/articles/10.3389/fnagi. 2021.815361/full\#supplementary-material

Parkinson-associated protein particles? Acta Neuropathol. 128, 463-476. doi: 10.1007/s00401-014-1324-9

Béringue, V., Vilotte, J. L., and Laude, H. (2008). Prion agent diversity and species barrier. Vet. Res. 39, 47. doi: 10.1051/vetres:2008024 
Bloom, G. S. (2014). Amyloid- $\beta$ and Tau: The trigger and bullet in alzheimer disease pathogenesis. JAMA Neurol. 71, 505-508. doi: 10.1001/jamaneurol.2013.5847

Bruce, M. E., Will, R. G., Ironside, J. W., McConnell, I., Drummond, D., Suttie, A., et al. (1997). Transmissions to mice indicate that "new variant" CJD is caused by the BSE agent. Nature. 389, 498-501. doi: 10.1038/39057

Caughey, B., and Lansbury, P. T. (2003). Protofibrils, pores, fibrils, and neurodegeneration: separating the responsible protein aggregates from the innocent bystanders. Annu. Rev. Neurosci. 26, 267-298. doi: 10.1146/annurev.neuro.26.010302.081142

Chiti, Z., Knutsen, O. M., Betmouni, S., and Greene, J. R. T. (2006). An integrated, temporal study of the behavioural, electrophysiological and neuropathological consequences of murine prion disease. Neurobiol. Dis. 22, 363-373. doi: 10.1016/j.nbd.2005.12.002

Collinge, J. (2016). Mammalian prions and their wider relevance in neurodegenerative diseases. Nature. 217-26. doi: 10.1038/nature20415

Collinge, J., Sidle, K. C., Meads, J., Ironside, J., and Hill, A. F. (1996). Molecular analysis of prion strain variation and the aetiology of "new variant" CJD. Nature. 383, 685-690. doi: 10.1038/383685a0

Costassa, E. V., Fiorini, M., Zanusso, G., Peletto, S., Acutis, P., and Baioni, E. (2016). Characterization of amyloid- $\beta$ deposits in bovine brains. J. Alzheimer's Dis. 51, 875. doi: 10.3233/JAD-151007

Duran-Aniotz, C., Morales, R., Moreno-Gonzalez, I., Hu, P. P., Fedynyshyn, J., and Soto, C. (2014). Aggregate-depleted brain fails to induce abeta deposition in a mouse model of Alzheimer's disease. PLoS ONE. 9, e89014. doi: 10.1371/journal.pone.0089014

Duran-Aniotz, C., Morales, R., Moreno-Gonzalez, I., Hu, P. P., and Soto, C. (2013). Brains from non-Alzheimer's individuals containing amyloid deposits accelerate Abeta deposition in vivo. Acta. Neuropathol. Commun. 1, 76. doi: 10.1186/2051-5960-1-76

Duran-Aniotz, C., Moreno-Gonzalez, I., Gamez, N., Perez-Urrutia, N., VegasGomez, L., and Soto, C. (2021). Amyloid pathology arrangements in Alzheimer's disease brains modulate in vivo seeding capability. Acta. Neuropathol. Commun. 9, 1-13. doi: 10.1186/s40478-021-01155-0

Eisele, Y. S., Obermüller, U., Heilbronner, G., Baumann, F., Kaeser, S. A., and Wolburg, H. (2010). Peripherally applied Abeta-containing inoculates induce cerebral beta-amyloidosis. Science. 330, 980-982. doi: 10.1126/science.11 94516

Fernández-Borges, N., Eraña, H., Elezgarai, S. R., Harrathi, C., Gayosso, M., and Castilla, J. (2013). Infectivity versus seeding in neurodegenerative diseases sharing a prion-like mechanism. Int. J. Cell Biol. 2013, 583498. doi: $10.1155 / 2013 / 583498$

Fritschi, S. K., Langer, F., Kaeser, S. A., Maia, L. F., Portelius, E., and Pinotsi, D. (2014). Highly potent soluble amyloid-beta seeds in human Alzheimer brain but not cerebrospinal fluid. Brain. 137, 2909-2915. doi: 10.1093/brain/awu255

Gomez-Isla, T., Spires, T., and Calignon A. D., and Hyman, B. T. (2008). Neuropathology of Alzheimer's disease. Handb. Clin. Neurol. 89, 233-243. doi: $10.1016 /$ S0072-9752(07)01222-5

Huang, Y., and Mucke, L. (2012). Alzheimer mechanisms and therapeutic strategies. Cell. 148, 1204-1222. doi: 10.1016/j.cell.2012.02.040

Irwin, D. J., Abrams, J. Y., Schonberger, L. B., Leschek, E. W., Mills, J. L., and Lee, V. M. Y. (2013). Evaluation of potential infectivity of Alzheimer and Parkinson disease proteins in recipients of cadaver-derived human growth hormone. JAMA Neurol. 70, 462-468. doi: 10.1001/jamaneurol.2013.1933

Jankowsky, J. L., Fadale, D. J., Anderson, J., Xu, G. M., Gonzales, V., and Jenkins, N. A. (2004). Mutant presenilins specifically elevate the levels of the 42 residue beta-amyloid peptide in vivo: evidence for augmentation of a 42 -specific gamma secretase. Hum. Mol. Genet. 13, 159-170. doi: 10.1093/hmg/ddh019

Jaunmuktane, Z., Mead, S., Ellis, M., Wadsworth, J. D., Nicoll, A. J., and Kenny, J. (2015). Evidence for human transmission of amyloid-beta pathology and cerebral amyloid angiopathy. Nature. 525, 247-250. doi: 10.1038/nature15369

Johnstone, E. M., Chaney, M. O., Norris, F. H., Pascual, R., and Little, S. P. (1991). Conservation of the sequence of the Alzheimer's disease amyloid peptide in dog, polar bear and five other mammals by cross-species polymerase chain reaction analysis. Brain Res. Mol. Brain Res. 10, 299-305. doi: 10.1016/0169-328X(91)90088-F

Kane, M. D., Lipinski, W. J., Callahan, M. J., Bian, F., and Durham, R. (2000). Evidence for seeding of beta -amyloid by intracerebral infusion of Alzheimer brain extracts in beta -amyloid precursor protein-transgenic mice. J. Neurosci. 20, 3606-11doi: 10.1523/JNEUROSCI.20-10-03606.2000

Langer, F., Eisele, Y. S., Fritschi, S. K., Staufenbiel, M., Walker, L. C., and Jucker, M. (2011). Soluble Abeta seeds are potent inducers of cerebral beta-amyloid deposition. JNeurosci. 31, 14488-14495. doi: 10.1523/JNEUROSCI.3088-11.2011

Masters, C. L., and Selkoe, D. J. (2012). Biochemistry of amyloid-protein and amyloid deposits in Alzheimer disease. Cold Spring Harb. 2, a006262. doi: 10.1101/cshperspect.a006262

Meyer-Luehmann, M., Coomaraswamy, J., Bolmont, T., Kaeser, S., Schaefer, C., and Kilger, E. (2006). Exogenous induction of cerebral betaamyloidogenesis is governed by agent and host. Science. 313, 1781-1784. doi: $10.1126 /$ science.1131864

Morales, R., Bravo-Alegria, J., Duran-Aniotz, C., and Soto, C. (2015a). Titration of biologically active amyloid $-\beta$ seeds in a transgenic mouse model of Alzheimer's disease. Sci. Reports. 5, 1-8. doi: 10.1038/srep09349

Morales, R., Bravo-Alegria, J., Moreno-Gonzalez, I., Duran-Aniotz, C., Gamez, N., and Edwards, G. (2021). Transmission of cerebral amyloid pathology by peripheral administration of misfolded A $\beta$ aggregates. Mol. Psychiatry. 2021, 1-12. doi: 10.1038/s41380-021-01150-w

Morales, R., Callegari, K., and Soto, C. (2015b). Prion-like features of misfolded A $\beta$ and tau aggregates. Virus Res. 207, 106-112. doi: 10.1016/j.virusres.2014.12.031

Morales, R., Duran-Aniotz, C., Castilla, J., Estrada, L. D., and Soto, C. (2011). De novo induction of Amyloid-beta deposition in vivo. Mol. Psych. 17, 1347-53. doi: $10.1038 / \mathrm{mp} .2011 .120$

Moreno-Gonzalez, I., and Soto, C. (2011). Misfolded protein aggregates: mechanisms, structures and potential for disease transmission. Semin. Cell Dev. Biol. 22, 482-487. doi: 10.1016/j.semcdb.2011.04.002

Moreno-Gonzalez, I., and Soto, C. (2018). Natural animal models of neurodegenerative protein misfolding diseases. Curr. Pharm. Des. 18. doi: 10.2174/138161212799315768

Nisbet, R. M., Polanco, J. C., Ittner, L. M., and Götz, J. (2015). Tau aggregation and its interplay with amyloid- $\beta$. Acta Neuropathol. 3, 207-220. doi: 10.1007/s00401-014-1371-2

Price, J. L., McKeel D. W. Jr., Buckles, V. D., Roe, C. M., Xiong, C., Grundman $\mathrm{M}$, et al. (2009). Neuropathology of nondemented aging: presumptive evidence for preclinical Alzheimer disease. NeurobiolAging. 30, 1026-1036. doi: 10.1016/j.neurobiolaging.2009.04.002

Prusiner, S. B. (1998). The prion diseases. Brain Pathol. 8, 499-513. doi: 10.1111/j.1750-3639.1998.tb00171.x

Prusiner, S. B. (2012). Cell biology. A unifying role for prions in neurodegenerative diseases. Science. 336, 1511-1513. doi: 10.1126/science.1222951

Rising, A., Gherardi, P., Chen, G., Johansson, J., Oskarsson, M. E., and Westermark, G. T. (2021). AA amyloid in human food chain is a possible biohazard. Sci Reports. 11, 21069. doi: 10.1038/s41598-02100588-w

Ritchie, D. L., Adlard, P., Peden, A. H., Lowrie, S., Le Grice, M., and Burns, K. (2017). Amyloid- $\beta$ accumulation in the CNS in human growth hormone recipients in the UK. Acta. Neuropathol. 134, 221-240. doi: 10.1007/s00401-017-1703-0

Rosen, R. F., Fritz, J. J., Dooyema, J., Cintron, A. F., Hamaguchi, T., and Lah, J. J. (2011). Exogenous seeding of cerebral beta-amyloid deposition in betaAPP-transgenic rats. J. Neurochem. 120, 660-666 doi: 10.1111/j.1471-4159.2011.07551.x

Scott, M. R., Will, R., Ironside, J., Nguyen, H. O., Tremblay, P., DeArmond, S. J., et al. (1999). Compelling transgenetic evidence for transmission of bovine spongiform encephalopathy prions to humans. Proc Natl Acad Sci U S A. 96, 15137-15142. doi: 10.1073/pnas.96.26.15137

Soto, C. (2003). Unfolding the role of protein misfolding in neurodegenerative diseases. Nat. Rev. Neurosci. 4, 49-60 doi: 10.1038/nrn1007

Soto, C. (2012). Transmissible proteins: expanding the prion heresy. Cell. 149, 968-77. doi: 10.1016/j.cell.2012.05.007

Soto, C., Estrada, L., and Castilla, J. (2006). Amyloids, prions and the inherent infectious nature of misfolded protein aggregates. Trends Biochem. Sci. 31, 150-155. doi: 10.1016/j.tibs.2006.01.002

Stöhr, J., Watts, J. C., Mensinger, Z. L., Oehler, A., Grillo, S. K., DeArmond, S. J., et al. (2012). Purified and synthetic Alzheimer's amyloid beta (A $\beta$ ) prions. Proc. Natl. Acad. Sci. U S A. 109 11025-30. doi: 10.1073/pnas.1206555109 
Tagliavini, F., Giaccone, G., Frangione, B., and Bugiani, O. (1988). Preamyloid deposits in the cerebral cortex of patients with Alzheimer's disease and nondemented individuals. NeurosciLett. 93, 191-196. doi: 10.1016/0304-3940(88)90080-8

Thal, D. R., Attems, J., and Ewers, M. (2014). Spreading of amyloid, tau, and microvascular pathology in alzheimer's disease: Findings from neuropathological and neuroimaging studies. J. Alzheimer's Dis. S421-S429. doi: 10.3233/JAD-141461

Walker, L. C., and Jucker, M. (2015). Neurodegenerative diseases: expanding the prion concept. Annu. Rev. Neurosci. 38, 87-103. doi: 10.1146/annurev-neuro-071714-033828

Walker, L. C., Levine, 3rd H., Mattson, M. P., and Jucker, M. (2006). Inducible proteopathies. Trends Neurosci. 29, 438-443. doi: 10.1016/j.tins.2006. 06.010

Watts, J. C., Giles, K., Grillo, S. K., Lemus, A., DeArmond, S. J., Prusiner, S. B. (2011). Bioluminescence imaging of Abeta deposition in bigenic mouse models of Alzheimer's disease. Proc. Natl. Acad. Sci. U S A. 108, 2528-2533. doi: $10.1073 /$ pnas. 1019034108
Conflict of Interest: The authors declare that the research was conducted in the absence of any commercial or financial relationships that could be construed as a potential conflict of interest.

Publisher's Note: All claims expressed in this article are solely those of the authors and do not necessarily represent those of their affiliated organizations, or those of the publisher, the editors and the reviewers. Any product that may be evaluated in this article, or claim that may be made by its manufacturer, is not guaranteed or endorsed by the publisher.

Copyright (C) 2022 Moreno-Gonzalez, Edwards, Morales, Duran-Aniotz, Escobedo, Marquez, Pumarola and Soto. This is an open-access article distributed under the terms of the Creative Commons Attribution License (CC BY). The use, distribution or reproduction in other forums is permitted, provided the original author(s) and the copyright owner(s) are credited and that the original publication in this journal is cited, in accordance with accepted academic practice. No use, distribution or reproduction is permitted which does not comply with these terms. 